\title{
The cause of parasitic infection in natural populations of Daphnia (Crustacea: Cladocera): the role of host genetics
}

\author{
Tom J. Little* and Dieter Ebert \\ Institut für Zoologie, Universität Basel, Rheinsprung 9, CH-4051 Basel, Switzerland
}

\begin{abstract}
Disease patterns in nature may be determined by genetic variation for resistance or by factors, genetic or environmental, which influence the host-parasite encounter rate. Elucidating the cause of natural infection patterns has been a major pursuit of parasitologists, but it also matters for evolutionary biologists because host resistance genes must influence the expression of disease if parasite-mediated selection is to occur. We used a model system in order to disentangle the strict genetic component from other causes of infection in the wild. Using the crustacean Daphnia magna and its sterilizing bacterial parasite Pasteuria ramosa, we tested whether genetic variation for resistance, as determined under controlled conditions, accounted for the distribution of infections within natural populations. Specifically, we compared whether the clonally produced great-granddaughters of those individuals that were infected in field samples (but were subsequently 'cured' with antibiotics) were more susceptible than were the great- granddaughters of those individuals that were healthy in field samples. High doses of parasite spores led to increased infection in all four study populations, indicating the importance of encounter rate. Host genetics appeared to be irrelevant to natural infection patterns in one population. However, in three other populations hosts that were healthy in the field had greater genetic-based resistance than hosts that were infected in the field, unambiguously showing the effect of host genetic factors on the expression of disease in the wild.
\end{abstract}

Keywords: infection; selection; parasite; resistance; genetic variation; coevolution

\section{INTRODUCTION}

Understanding the cause (genetic or environmental) of parasitic infection has clear utilitarian value because, if genes matter, their distribution may determine the incidence and severity of disease (e.g. Gilbert et al. 1998; Williams-Blangero et al. 1999). In addition, parasitemediated natural selection is at the heart of evolutionary hypotheses for explaining a number of important phenomena, including the maintenance of sexuality (Haldane 1949; Levin 1975; Jaenike 1978; Hamilton 1980) and the striking polymorphism of the vertebrate immune system (Hedrick 1994). Genetic variation for resistance must influence the expression of disease for parasitemediated natural selection to occur. However, in some studies genetic variation for resistance, although apparent during controlled exposure, was a poor predictor of either disease patterns in the field (Scott 1991) or the response to selection (Burdon \& Thompson 1995; Henter \& Via 1995), indicating that natural levels of environmental complexity can greatly reduce the significance of genes.

When comparing between infected and uninfected hosts in field samples it is normally difficult to use the controlled laboratory techniques necessary for separating the strict genetic component of resistance from factors influencing either the host-parasite encounter rate or condition-based effects which may make a host more susceptible when encounters occur (Bundy \& Medley 1992; Chan et al. 1994; Sorci et al. 1997; Little \& Ebert 1999). We overcame this problem with a model system

\footnotetext{
*Author and address for correspondence: Institute for Cell, Animal and Population Biology, University of Edinburgh, Kings Buildings, West Mains Road, Edinburgh EH9 3JT, UK (tom.little@ed.ac.uk).
}

that allowed us to test whether genetic variation for resistance, as determined under controlled conditions, accounted for the distribution of infections within natural populations. We studied the crustacean Daphnia magna and its horizontally transmitted bacterial parasite Pasteuria ramosa. Two features in particular make this system suitable. First, Daphnia can be clonally propagated so that hosts can be collected from the field and their genotypes remain unchanged while in the laboratory. Similarly, field samples of P.ramosa can be stored unchanged in the freezer until needed. It is thus possible to obtain a genetic 'snapshot' of both the host and parasite populations. Second, infected Daphnia can be 'cured' with antibiotics and then cultured normally so that it is possible to have the infected class of individuals represented in experiments.

\section{EXPERIMENTAL OVERVIEW}

Figure 1 summarizes the experimental design. We isolated infected and uninfected female hosts from four natural populations, each from a different pond. Infected individuals were cured and then all isolates were propagated clonally under controlled laboratory conditions for at least three generations in order to remove the effects of antibiotic treatment and control for maternal and grandmaternal effects across host lines. The reduction of maternal effects is critical for an unequivocal demonstration that differences between treatment groups stem from genetic differences (Falconer 1989). Following these generations, newborns were isolated and exposed to two or three different concentrations of parasite spores. Each population was studied separately and hosts were only 

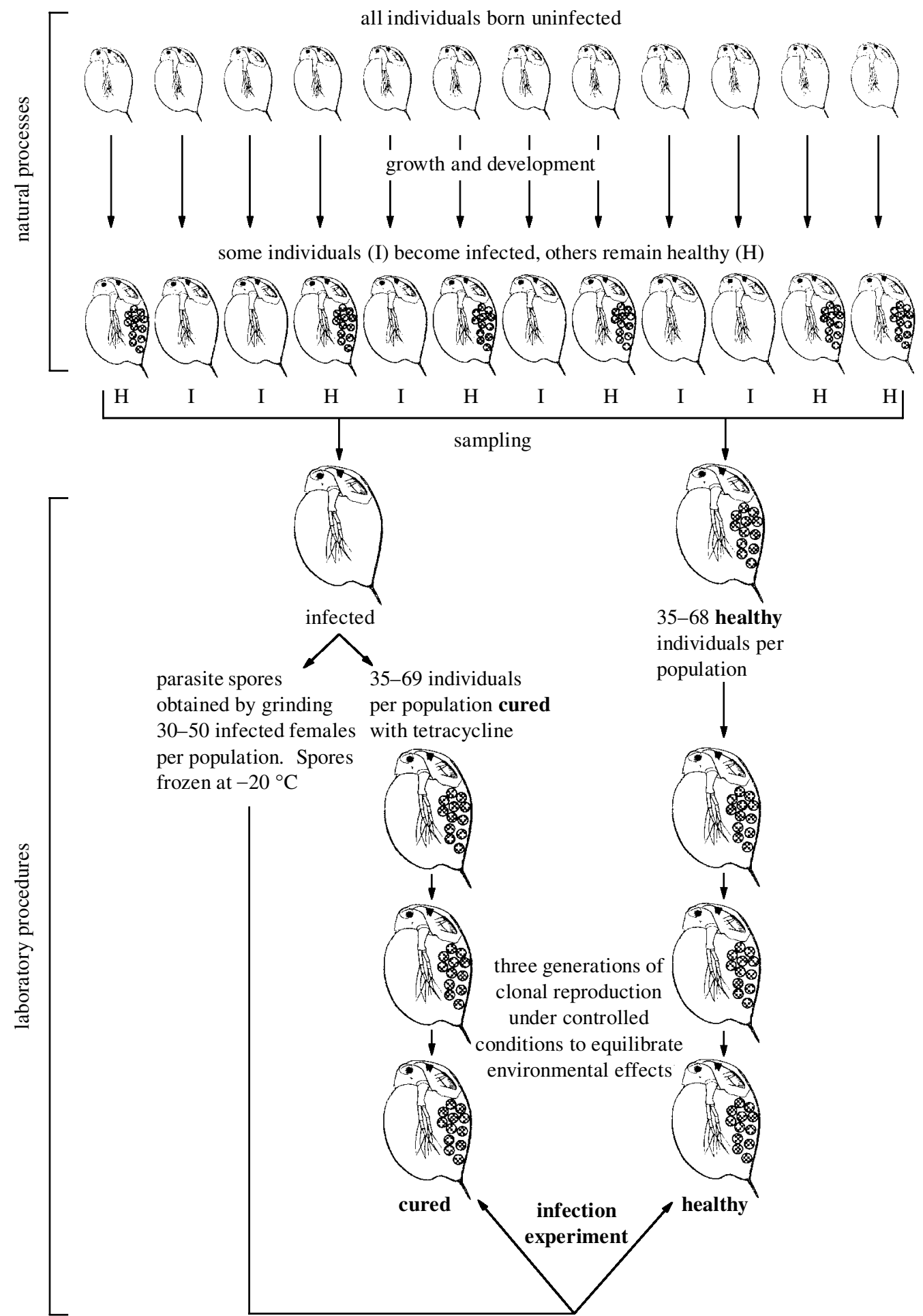

Figure 1. Overview of the design of experiments with the bacterium P. ramosa and its host D. magna. Pasteuria ramosa is a horizontally transmitted parasite and, thus, infections observed in field samples originated sometime between host birth and the time of collection (a period of approximately one to two weeks). In the wild, infected hosts are sterilized (and, thus, are depicted having no eggs in the brood chamber) and do not contribute offspring to subsequent generations. Experiments tested whether naturally occurring infections had a genetic basis.

exposed to parasites from their own pond. Each infection experiment compared the susceptibility of those 'genetic individuals' (clones) which were naturally infected (but had since been cured with antibiotics) with those that were naturally healthy at the time of collection.

\section{METHODS}

Hosts were collected from four ponds in northern Germany (each named after the closest village or estate, i.e. Rixdorf, Neudorf, Gaarzerfeld and Kniphagen) infected with P. ramosa. 
Each population was studied separately (i.e. experiments only exposed hosts to parasites from their own pond). Based on electrophoretic studies of 16 allozyme loci, each population was genetically diverse with between two and five polymorphic loci per population and no deviations from genetic equilibria. Specimens in field samples were classified as infected or uninfected based on the visible parasite spore mass in the haemolymph of infected individuals (see Ebert et al. 1996). Clonal lines of Daphnia found to be uninfected at the time of collection (these Daphnia are referred to as 'healthy') were established by placing single females in $100 \mathrm{ml}$ jars with synthetic pond water and fed regularly with an algal suspension (culture conditions as in Ebert et al. (1998)). Because P. ramosa sterilizes its host, in order to establish clonal lines of Daphnia infected at the time of collection we treated infected individuals in water containing $0.025 \mathrm{mg} \mathrm{ml}^{-1}$ tetracycline. After one to two weeks of treatment, antibiotic-treated Daphnia released a clutch of eggs into the brood chamber. At this stage the 'cured' Daphnia were placed in fresh water containing no tetracycline. When newborns were released, they were transferred to fresh water and clonally propagated in the same manner as healthy Daphnia.

Parasite spores for the experiments were collected by grinding wild-caught, infected females that had been kept in $100 \mathrm{ml}$ of water and fed well until they died. The resulting spore suspension contained spores derived from at least 30 wildcaught hosts. Spore concentrations were determined by counting spores in a bacterial counting chamber and diluting accordingly so that $20 \mu \mathrm{l}$ of spore solution were added for each spore dose used in the infection experiments.

In order to equilibrate the environmental effects among hosts prior to experiments, all isolates were maintained singly in $100 \mathrm{ml}$ of water and fed a constant amount of food $\left(3.75 \times 10^{6}\right.$ algal cells $\left.\mathrm{d}^{-1}\right)$. Each new generation was seeded with one newborn per Daphnia from the third clutch. Newborns from the third clutch of the third generation were used in the infection experiment. Infection experiments compared the susceptibility of healthy and cured Daphnia. Following a split-brood design, individual offspring from each Daphnia were placed singly into plastic test tubes containing $5 \mathrm{ml}$ of water (control) or $5 \mathrm{ml}$ of water and $20 \mathrm{ml}$ of parasite spores (treatments). There were two different spore dose treatments for population 4 (Kniphagen) and three different spore dose treatments for the other populations. After a five-day infection period, Daphnia were transferred to $100 \mathrm{ml}$ of water. We used a range of spore dose treatments in order to ensure moderate infection levels that were amenable to statistical analyses. The experiment with the Kniphagen population was repeated using a single spore dose and less food $\left(2.5 \times 10^{6}\right.$ cells ml $\left.^{-1}\right)$, but otherwise identical methods. Each experiment was run until no further infections were recorded, during which time the Daphnia were transferred to fresh water each time they produced a clutch. For those Daphnia that became infected and, hence, produced no clutches, transfers occurred every three to four days.

The trait of interest for all experiments was infectivity. This is a binary trait and was analysed with logistic regression. Infection history (cured or healthy) and spore dose were fitted together as explanatory variables in the GENMOD procedure of the SAS statistical package (link $=$ LOGIT, dist $=$ BIN and type 3 analysis) (SAS Institute, Inc. 1993).

In order to test whether the tetracycline treatment itself had any lasting effects on infectivity, we performed a separate experiment on nine randomly selected clones from the Gaarzerfeld population which were known to differ at allozyme loci. We

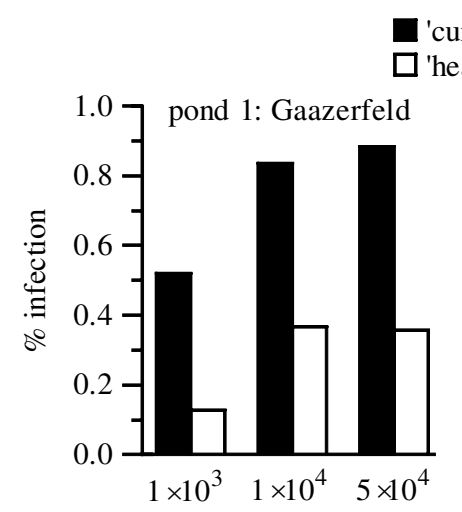

'cured'

'healthy'
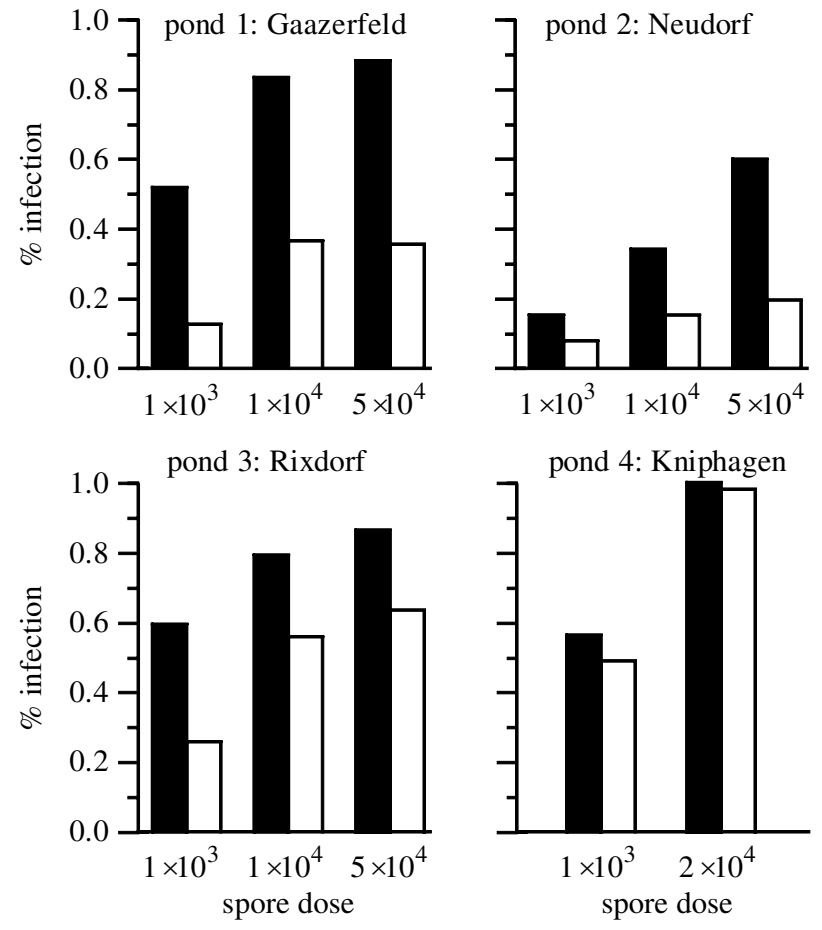

Figure 2. Results of infection experiments that compared the susceptibility of D. magna that were infected at the time of collection (but were cured with antibiotics prior to experiments) with the susceptibility of D. magna that were healthy at the time of collection. Four populations were studied and infections were carried out with two or three parasite spore doses. Populations 1-3 (Gaarzerfeld, Neudorf and Rixdorf) showed significant differences between cured and healthy isolates, while population 4 (Kniphagen) showed no difference (see table 1).

produced 30 replicates of each these clones and 15 of these were used as a 'no tetracycline' treatment, while the other 15 were exposed to tetracycline for three weeks. Following tetracycline exposure, all replicates were maintained under controlled conditions for two generations. Newborns were then used in an infection experiment that compared the susceptibility of those replicates that had previously experienced tetracycline with those that had not. The protocols were similar to those used in the main experiments. The results were analysed as above with 'clone' and 'tetracycline' fitted together as explanatory variables in a logistic regression. The analysis indicated that the clones differed markedly in their levels of infection $\left(\chi^{2}=85.7\right.$, d.f. $=5$ and $p<0.0001)$, but no effect of tetracycline treatment was detected (proportion infected in no tetracycline treatment $=$ 0.59 , proportion infected in tetracycline treatment $=0.66$ and total sample size $=169)\left(\chi^{2}=1.2\right.$, d.f. $=1$ and $\left.p<0.27\right)$.

\section{RESULTS AND DISCUSSION}

Infection status in the field explained the differences in susceptibility among individuals under controlled laboratory conditions in three of the four populations (figure 2 and table 1). In these three populations, clonal offspring of Daphnia that were naturally healthy were more resistant than those which were infected at the time of collection and this resistance has a genetic basis. By showing that infection naturally affected the genetic 
Table 1. Likelihood ratio statistics for the results of infection experiments that compared the suscep tibility of D. magna that were infected at the time of collection (but were cured with antibiotics prior to experiments) with the susceptibility of D. magna that were healthy at the time of collection

\begin{tabular}{|c|c|c|c|c|c|c|c|c|}
\hline \multirow[b]{2}{*}{ population } & \multicolumn{2}{|c|}{ sample size } & \multicolumn{3}{|c|}{ infection history } & \multicolumn{3}{|c|}{ spore dose } \\
\hline & cured & healthy & $\chi^{2}$ & d.f. & $p$ & $\chi^{2}$ & d.f. & $p$ \\
\hline Gaarzerfeld & 41 & 46 & 65.0 & 1 & $<0.0001$ & 25.4 & 2 & $<0.0001$ \\
\hline Neudorf & 68 & 63 & 26.2 & 1 & $<0.0001$ & 24.1 & 2 & $<0.0001$ \\
\hline Rixdorf & 50 & 61 & 25.8 & 1 & $<0.0001$ & 29.4 & 2 & $<0.0001$ \\
\hline Kniphagen & 49 & 41 & 2.0 & 1 & n.s. & 64.2 & 1 & $<0.0001$ \\
\hline
\end{tabular}

Table 2. The estimated infective dose (number of spores) required to infect $50 \%$ of hosts $\left(\mathrm{ID}_{50}\right.$ ) differed markedly between D. magna that were infected at the time of collection and D. magna that were healthy at the time of collection in three of the four populations (see figure 1)

(By weighting $\mathrm{ID}_{50}$ s with the parasite prevalence (the proportion of individuals infected in field samples) it is possible to estimate the population average $\mathrm{ID}_{50}$ at the time of collection. Assuming only those individuals in the healthy class will reproduce (P. ramosa sterilizes its host), the population $\mathrm{ID}_{50}$ following selection will be equal to the $\mathrm{ID}_{50}$ of healthy Daphnia. The last column thus represents an estimated change in the population $\mathrm{ID}_{50}$ due to selection by parasites. ID $_{50}$ s were calculated by logistic regression of experimental infectivity and spore dose (SAS procedure PROBIT) (SAS Institute, Inc. 1990). The confidence limits around $\mathrm{ID}_{50}$ estimates were large and could not be calculated in all cases.)

\begin{tabular}{|c|c|c|c|c|c|}
\hline \multirow[b]{2}{*}{ population } & \multicolumn{2}{|c|}{$\mathrm{ID}_{50} \times 10^{3}$} & \multirow{2}{*}{$\begin{array}{l}\text { percentage } \\
\text { parasite } \\
\text { prevalence }\end{array}$} & \multirow{2}{*}{$\begin{array}{l}\text { estimated population average } \\
\text { before selection }\left(\times 10^{3}\right)\end{array}$} & \multirow{2}{*}{$\begin{array}{c}\text { estimated percentage } \\
\text { change in } \text { ID }_{50} \text { after } \\
\text { selection }\end{array}$} \\
\hline & cured & healthy & & & \\
\hline Gaarzerfeld & 0.4 & 9 & 24 & 6.9 & 30.0 \\
\hline Neudorf & 2.6 & 8500 & 52 & 4100.0 & 107.6 \\
\hline Rixdorf & 0.8 & 190 & 11 & 170.0 & 12.3 \\
\hline Kniphagen & 0.9 & 1 & 7 & 1.0 & 0.4 \\
\hline
\end{tabular}

subset of the population which is indeed more susceptible, these results exclude a variety of confounding factors and provide an unequivocal linkage between genetic variation for resistance and the natural occurrence of infection.

This result has important implications for the occurrence of parasite-mediated selection because, for selection to occur, genetic variation for resistance must penetrate the environmental noise of natural systems and be a significant cause of variation in infection. As only healthy females can reproduce (females infected with P. ramosa are sterilized) the genetic differences observed very probably represent fitness differences in nature. In order to gain insight into the response to selection which might follow from these patterns of genetic variation, we calculated the infective dose (number of spores) required to infect 50\% of hosts $\left(\mathrm{ID}_{50}\right)$ and then estimated how this might change over one generation. This analysis (table 2) indicated that changes in a population's average resistance might be very large, with the population ID $_{50}$ increasing by as much as twofold after selection. Based on the generation time of D. magna and the developmental time of P. ramosa, the time between infection and our sampling would be from one to four weeks. This evidence for natural selection and rapid evolution (for a comparison see Thompson 1998) suggests that coevolutionary interactions may proceed on remarkably brief time-scales.

There may of course be constraints on selection and these might in turn influence the maintenance of genetic variation in this system. For example, resistance might be traded off against other fitness components (antagonistic pleiotropy) and this might slow the response to selection. Although we cannot rule out a cost of resistance, one was not apparent in our studies as comparisons between hosts in the control groups showed that individuals in the cured class did not differ significantly from those in the healthy class with regard to life-history characters (T. J. Little and D. Ebert, unpublished data). Alternatively, the maintenance of genetic variation in this system could be explained by ongoing, frequency-dependent selection where parasites quickly specialize on common host genotypes, thereby generating a selective advantage for rarer genotypes (Dybdahl \& Lively 1995). The maintenance of genetic variation through this process may depend on the underlying genetic control of resistance (Clay \& Kover 1996) and studies for uncovering the nature of hostparasite specificities in this system would be valuable. In addition, future work ought to include time-series studies designed to record the magnitude of genetic change directly.

Spore dose effects were highly significant $(p<0.001)$ in all populations (figure 2 and table 1). However, for one population (Kniphagen) the spore dose effects explained all the variation in infection and there was no evidence that host genetics were a cause of disease patterns. We repeated the experiment on this population, but under low food conditions in order to test whether stressing the hosts might illuminate genetic differences. However, the outcome of this second experiment confirmed the result of the first experiment (infection level, cured $=0.71$ and healthy $=0.72)\left(\chi^{2}=0.64\right.$, d.f. $=1$ and n.s. $)$. We further 


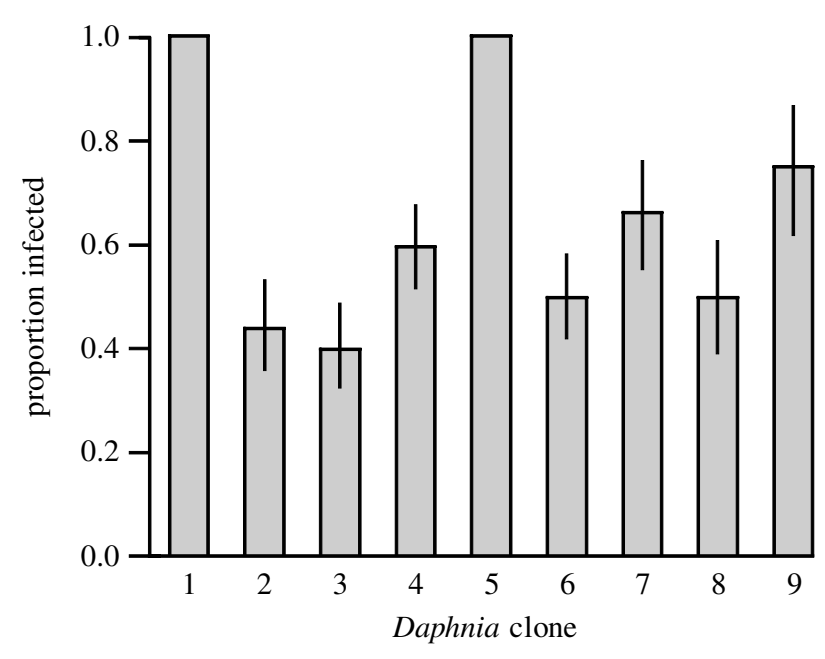

Figure 3. Variation in infectivity among nine allozymically distinct clones of D. magna challenged with 1000 spores $\mathrm{ml}^{-1}$ of the bacterium $P$. ramosa. Ten replicates (minus those which died during the experiment) of each clone were compared in a log-linear model (SAS procedure CATMOD with total sample size $=74)\left(\right.$ d.f. $=8, \chi^{2}=26.2$ and $\left.p<0.001\right)$. The infection procedures were identical to those used in the main experiments. Bars are standard errors.

tested whether the Kniphagen population harboured genetic variation for infectivity irrespective of infection history. We measured the infectivity of each of nine randomly selected clones which differed at allozyme loci and the results indicated the presence of clonal variation (figure 3). Why clonal variation for infectivity was apparently irrelevant to natural infection patterns is unclear. Numerous explanations are plausible, including genotype $\times$ environment interactions or the distribution of disease in this population being best explained by nongenetic factors. This result reinforces the need for confirming laboratory-based heritability measurements with field data and highlights the importance of distinguishing between factors influencing encounter rates and those influencing resistance once encounters occur.

Past studies have revealed genetic variation for resistance in controlled experiments (Jarosz \& Burdon 1991; Thompson \& Burdon 1992; Ebert et al. 1998; Webster \& Woolhouse 1998; Carroll et al. 2000) or have shown that disease tends to congregate around particular genotypes or families (Grosholz 1994; Dybdahl \& Lively 1995; Little \& Ebert 1999; Smith et al. 1999; Williams-Blangero et al. 1999). However, predicting and showing a response to selection based on patterns of genetic variation appears to be more difficult (Burdon \& Thompson 1995; Henter \& Via 1995; Little \& Ebert 1999). It may be that genetic variation, although detectable in the laboratory, is overwhelmed in noisy, natural environments, as might have been the case in our Kniphagen population and in other studies where genetic variation determined during controlled exposure appeared to bear little relevance to who becomes sick and who does not in nature (Scott 1991). However, ours is perhaps the first study capable of elucidating the strict genetic component of natural infection patterns and, for three populations, genetic differences for resistance were evident despite the presence of other genetic factors and the panoply of environmental factors which also influence the spread of disease. This result shows not only that resistance variation exists, but that it matters.

We thank Larry Weider, Gerhard Augustin and Jürgen Hottinger for their assistance during data collection. This work was supported by a Swiss-National Fond grant to D.E. and by a Schweizerische Akademie der Naturwissenschaften Reisestipendium from the Eidgenössische Technische Hockshule, Zürich, to T.J.L. We thank Martin Ackermann, Achim Carius, Brian Chan, Nick Colegrave, Michael Doebeli, Tad Kawecki, Paula Kover, Margaret MacKinnon, Peter Müller, Josephine Pemberton, Andrew Read, Ian Sanders and Steve Stearns for comments and/or discussion.

\section{REFERENCES}

Bundy, D. A. P. \& Medley, G. F. 1992 Immuno-epidemiology of human geohelminthiasis: ecological and immunological determinants of worm burden. Parasitology 104, S105-S119.

Burdon, J. J. \& Thompson, J. N. 1995 Changed patterns of resistance in a population of Linum marginale attacked by the rust pathogen Melampspora lini. F. Ecol. 83, 199-206.

Carroll, M. J., Zangerl, A. R. \& Berenbaum, M. R. 2000 Heritability estimates for octyl acetate and octyl butyrate in the mature fruit of the wild parsnip. 7. Hered. 91, 68-71.

Chan, L., Bundy, D. A. P. \& Kan, S. P. 1994 Genetic relatedness as a determinant of predisposition to Ascaris lumbricoides and Trichuris trichiura infection. Parasitology 108, 77-80.

Clay, K. \& Kover, P. 1996 The red queen hypothesis and plant/ pathogen interactions. A. Rev. Phytopathol. 34, 29-50.

Dybdahl, M. F. \& Lively, C. M. 1995 Host-parasite interactions: infection of common clones in natural populations of a freshwater snail (Potamopyrgus antipodarum). Proc. R. Soc. Lond. B 260, 99-103.

Ebert, D., Rainey, P., Embley, T. M. \& Scholz, D. 1996 Development, life cycle, ultrastructure and phylogenetic position of Pasteuria ramosa Metchnikoff 1888: rediscovery of an obligate endoparasite of Daphnia magna Straus. Phil. Trans. R. Soc. Lond. B 351, 1689-1701.

Ebert, D., Zschokke-Rohringer, C. D. \& Carius, H. J. 1998 Within and between population variation for resistance of Daphnia magna to the bacterial endoparasite Pasteuria ramosa. Proc. R. Soc. Lond. B 265, 2127-2134.

Falconer, D. S. 1989 Quantitative genetics. Essex, UK: Longman.

Gilbert, S. C., Plebanski, M., Gupta, S., Morris, J., Cox, M., Aidoo, M., Kwiatkowski, D., Greenwood, B. M., Whittle, H. C. \& Hill, A. V. S. 1998 Association of malaria parasite population structure, HLA, and immunological antagonism. Science 279, 1173-1177.

Grosholz, E. D. 1994 The effects of host genotype and spatial distribution on trematode parasitism in a bivalve population. Evolution 48, 1514-1524.

Haldane, J. B. S. 1949 Disease and evolution. Suppl. Anno 19, 68-75.

Hamilton, W. D. 1980 Sex versus non-sex versus parasite. Oikos 35, 282-290.

Hedrick, P. W. 1994 Evolutionary genetics of the major histocompatibility complex. Am. Nat. 143, 945-964.

Henter, H. J. \& Via, S. 1995 The potential for coevolution in a host-parasitoid system. I. Genetic variation within an aphid population in susceptibility to a parasitic wasp. Evolution $\mathbf{4 9}$, 427-438.

Jaenike, J. 1978 A hypothesis to account for the maintenance of sex within populations. Evol. Theor. 3, 191-194.

Jarosz, A. M. \& Burdon, J. J. 1991 Host-pathogen interactions in natural populations of Linum marginale and Melampsora lini: II. Local and regional variation in patterns of resistance and racial structure. Evolution 45, 1618-1627. 
Levin, D. 1975 Pest pressure and recombination systems in plants. Am. Nat. 109, 437-451.

Little, T. J. \& Ebert, D. 1999 Associations between parasitism and host genotype in natural populations of Daphnia (Crustacea: Cladocera). 7. Anim. Ecol. 67, 134-149.

SAS Institute, Inc. 1990 SAS/STAT, v. 6.06. Cary, NC: SAS Institute, Inc.

SAS Institute, Inc. 1993 SAS technical report P-243, SAS/STAT software: the GENMOD procedure, release 6.09. Cary, NC: SAS Institute, Inc.

Scott, M. E. 1991 Heligmosomoides polygrus (Nematoda): susceptible and resistant strains are indistinguishable following natural infection. Parasitology 103, 429-438.

Smith, J. A., Wilson, K., Pilkington, J. G. \& Pemberton, J. M. 1999 Heritable variation in resistance to gastro-intestinal nematodes in an unmanaged mammal population. Proc. $R$. Soc. Lond. B 266, 1283-1290.

Sorci, G., Møller, A. P. \& Boulinier, T. 1997 Genetics of hostparasite interactions. Trends Ecol. Evol. 12, 196-200.

Thompson, J. N. 1998 Rapid evolution as an ecological process. Trends Ecol. Evol. 13, 329-332.

Thompson, J. N. \& Burdon, J. J. 1992 Gene-for-gene coevolution between plants and parasites. Nature 360, 121-125.

Webster, J. P. \& Woolhouse, M. E. J. 1998 Selection and strain specificity of compatibility between snail intermediate hosts and their parasitic schistosomes. Evolution 52, 1627-1634.

Williams-Blangero, S., Subedi, J., Upadhayay, R. P., Manral, D. B., Rai, D. R., Jha, B., Robinson, E. S. \& Blangero, J. 1999 Genetic analysis of susceptibility to infection with Ascaris lumbricoides. Am. F. Trop. Med. Hyg. 60, 921-926. 